\title{
Role of race and socioeconomic status in transplant access
}

Racial disparities exist in various stages of the transplantation process, including referral, evaluation, waitlisting and organ receipt. Increasing evidence suggests that socioeconomic status (SES) might also influence transplant access. Two studies that examine racial and socioeconomic disparities have now been published in the American Journal of Transplantation.

\section{4 ...significantly fewer black patients proceeded in starting the evaluation, waitlisting and receiving a transplant 77}

In the first study, the effects of race and SES on early steps of the transplantation process were examined in 2,291 patients referred for transplant evaluation at the Emory Transplant Center, based in Southeastern USA. "Working at one of the largest transplant centers in the USA, we have a unique opportunity to answer some of the questions that this early research raised", says Rachel Patzer. "There are several unique aspects to this region related to our research. First, there is a higher concentration of poverty in the south versus north of the USA. Second, the racial distribution is different here compared with the rest of the country."

The researchers found that significantly fewer black patients proceeded in starting the evaluation, waitlisting and receiving a transplant. In addition, the duration of time that patients remained in each transplant step was longer for black patients than for white patients, with the greatest difference observed in the final step, waitlisting to transplantation. SES factors explained $30.6 \%$ of the reduced rate of transplantation among black versus white patients. However, racial disparities persisted after adjustment for SES.

In the second study, the interplay between race and SES was examined in a cohort of 8,452 pediatric patients with end-stage renal disease. To determine whether SES explained the impact of race on access to kidney transplantation, the researchers sequentially adjusted for clinical, demographic and SES factors. As in the first study, the rate of transplantation was lower among Hispanic and black patients than among white patients. Disparities were greatest among patients aged 18-20 years; in this age group, the rate of transplantation was $40 \%$ lower in black patients and $38 \%$ lower in Hispanics than in white patients. Adjusting for SES had little effect on these results. "Further research is needed to identify what may be explaining the racial disparities that still exist in access to kidney transplantation," comments Patzer. "We hypothesize this may have something to do with transition of care from pediatric to adult transplant centers or from other personal and/or family changes such as less adult supervision, changes in health insurance benefits, or other factors." Although SES factors may mitigate some of the racial disparities observed in kidney transplantation access, much of the racial disparity remains unexplained.

Helene Myrvang

Original articles Patzer, R. E. et al. The role of race and poverty on steps to kidney transplantation in the Southeastern United States. Am. J. Transplant. doi:10.1111/j.1600-6143.2011.03927.x | Patzer, R. E. et al. Racial disparities in pediatric access to kidney transplantation: does socioeconomic status play a role? Am. J. Transplant. doi:10.1111/j.16006143.2011.03888.x 